\title{
Desmin splice variants causing cardiac and skeletal myopathy
}

Kye-Yoon Park, Marinos C Dalakas, Hans H Goebel, Victor J Ferrans, Christina

Semino-Mora, Svetlana Litvak, Kazuyo Takeda, Lev G Goldfarb

\begin{abstract}
Desmin myopathy is a hereditary or sporadic cardiac and skeletal myopathy characterised by intracytoplasmic accumulation of desmin reactive deposits in muscle cells. We have characterised novel splice site mutations in the gene desmin resulting in deletion of the entire exon 3 during the pre-mRNA splicing. Sequencing of cDNA and genomic DNA identified a heterozygous de novo $A$ to $G$ change at the +3 position of the splice donor site of intron 3 (IVS $3+3 A \rightarrow G)$ in a patient with sporadic skeletal and cardiac myopathy. A G to A transition at the highly conserved -1 nucleotide position of intron 2 affecting the splice acceptor site (IVS2-1G $\rightarrow$ A) was found in an unrelated patient with a similar phenotype. Expression of genomic DNA fragments carrying the IVS $3+3 \mathrm{~A} \rightarrow \mathrm{G}$ and IVS2-1G $\rightarrow A$ mutations confirmed that these mutations cause exon 3 deletion. Aberrant splicing leads to an in frame deletion of 32 complete codons and is predicted to result in mutant desmin lacking 32 amino acids from the 1B segment of the alpha helical rod. Functional analysis of the mutant desmin in SW13 (vim-) cells showed aggregation of abnormal coarse clumps of desmin positive material dispersed throughout the cytoplasm. This is the first report on the pathogenic potentials of splice site mutations in the desmin gene.

(f Med Genet 2000;37:851-857)
\end{abstract}

Keywords: cardiac and skeletal myopathy; desmin splice site mutations; expression study; genotype-phenotype correlation

Neuropathology, Mainz

University Medical

Centre, D-55131

Mainz, Germany

H H Goebel

Pathology Section, National Heart, Lung, and Blood Institute,

NIH, Bethesda, MD

20892, USA

V J Ferrans

K Takeda

Correspondence to: Dr Goldfarb, National Institutes of Health, Bldg 10, Room 4B37, 10 Center Drive, MSC 1361, Bethesda Maryland 20892-1361, USA, goldfarb@codon.nih.gov

Revised version received 20 May 2000

Accepted for publication

3 July 2000

Desmin myopathy is a heterogeneous group of inherited or sporadic disorders presenting with progressive limb muscle weakness spreading to truncal, neck flexor, facial, bulbar, and respiratory muscles. ${ }^{12}$ Skeletal myopathy is typically combined with cardiomyopathy manifested by cardiac conduction blocks, arrhythmias, and restrictive dysfunction. ${ }^{3}$ The illness progresses slowly, over one or two decades, leaving the patient extremely disabled and needing a wheelchair, pacemaker, or respirator. Sections of the affected skeletal muscle show abnormal fibre areas marked with amorphous material and particles, spherical hyaline structures, and small vacuoles. ${ }^{4}$ Immunostaining for desmin is positive in all regions showing the presence of amorphous or granular material. Abnormal accumulations of desmin attract other proteins, most commonly dystrophin, actin, gelsolin, and ubiquitin. ${ }^{5}$ Desmin is an intermediate filament protein of the heart, skeletal, and smooth muscle cells normally serving as a mechanical integrator of the contractile action. ${ }^{6}$ In striated muscle, desmin encircles and makes connections between neighbouring $\mathrm{Z}$ disks. In accordance with its function, a desmin molecule is organised into three domains: an extended alpha helical rod-like middle domain flanked by globular $\mathrm{N}$ - and C-terminal domains. ${ }^{7}$ The middle rod consists of four segments, $1 \mathrm{~A}, 1 \mathrm{~B}$, $2 \mathrm{~A}$, and $2 \mathrm{~B}$, interrupted by three linkers. ${ }^{8}$ The alpha helical segments of the well conserved rod domain maintain a seven residue (heptad) repeat pattern; this structure guides two identical polypeptides into formation of a double stranded coiled coil dimer by interchain twisting. ${ }^{9}$ In building an intermediate filament network, desmin interacts with other proteins which cross link desmin filaments and attach them to the membranes.

Desmin is encoded by a single copy gene (DES) located in the $2 \mathrm{q} 35$ band. ${ }^{10}$ The desmin gene has nine exons and is highly conserved among vertebrates. ${ }^{11}$ Growing and adult desmin knockout mice develop a multisystem disorder involving severe disruption of muscle architecture including loss of lateral alignment of myocytes and abnormal mitochondrial organisation. ${ }^{12}$ These abnormalities are most severe in the myocardium, exhibiting disruption of sarcolemma, changes in the intercalated discs, necrosis, and extensive calcification. ${ }^{13}{ }^{14}$ The phenotypes observed in desmin deficient mice and the results of in vitro desmin assembly experiments suggest that mutant desmin is assembly incompetent ${ }^{1516}$ and capable of disrupting the pre-existing filament network in a dominant negative fashion. ${ }^{17}$

We have recently characterised three mis-

mutations in the desmin gene in patients with cardiac and skeletal myopathy. ${ }^{18}$ MunozMarmol et $a l^{19}$ described a disease associated 7 aa deletion in the $1 \mathrm{~B}$ segment of the desmin rod and showed the inability of the mutant desmin to produce an intermediate filament network. A mutation in the desmin tail domain was recently shown to cause cardiomyopathy with no signs of skeletal myopathy, ${ }^{20}$ and a missense L345P mutation was described in a large family with autosomal dominant myopathy. ${ }^{21}$

In the current study, we identified and characterised mutations occurring in the splice sites flanking exon 3 of the desmin gene. They result in skipping of exon 3 during pre-mRNA splicing and an in frame deletion of 32 amino acids (214 to 245) from the $1 \mathrm{~B}$ alpha helical 
subdomain of the mutant desmin protein. Immunofluorescent analysis of SW13 (vim-) cells transfected with mutant cDNA showed that desmin lacking these 32 amino acids accumulated into abnormal disrupted aggregates reminiscent of desmin deposits in the patients' affected muscles.

\section{Methods}

MUTATION ANALYSIS

RNA was extracted from muscle biopsy tissue obtained from a patient and a control using the $\mathrm{RNeasy}^{\mathrm{TM}}$ kit (Qiagen). Three micrograms of total RNA was used for reverse transcription (RT) performed according to the Superscript RT protocol (Gibco BRL). Full length cDNA was amplified by PCR with primers DIF (5'-CCGTCACCATGAGCC AGG-3') and DCR (5'-AGAGGGTCTCTCGTCTTTAG$\left.3^{\prime}\right)$. Amplification was carried out in a total volume of $20 \mu \mathrm{l}$ with $1 \mu \mathrm{l}$ of single stranded cDNA, $0.5 \mu \mathrm{mol} / 1$ of each primer, $125 \mu \mathrm{mol} / 1$ of each dNTP, $1.5 \mathrm{mmol} / 1$ of $\mathrm{MgCl}_{2}, 10 \mathrm{mmol} / 1$ Tris-HCl (pH 8.3), $50 \mathrm{mmol} / \mathrm{l}$ of $\mathrm{KCl}$, and 0.6 units of rTth DNA polymerase (Perkin Elmer). PCR cycling was performed under the following conditions: incubation for four minutes at $94^{\circ} \mathrm{C}$, processing through 35 cycles $(60 \mathrm{sec}-$ onds at $94^{\circ} \mathrm{C}, 60$ seconds at $48^{\circ} \mathrm{C}, 60$ seconds at $72^{\circ} \mathrm{C}$ ), and final incubation at $72^{\circ} \mathrm{C}$ for 10 minutes. After agarose gel electrophoresis, DNA bands were excised, eluted, purified by QiaEx II (Qiagen), cloned into the TA cloning vector (Invitrogen), and cycle sequenced in at least eight clones using DyeTerminator ${ }^{\mathrm{TM}}$ Sequencing Protocol (Perkin Elmer) on an automated ABI 373A sequencer.

Genomic DNA was extracted from anticoagulated blood using the Wizard ${ }^{\mathrm{TM}}$ Genomic DNA Purification Kit (Promega) and from muscle biopsy tissue, and used as template for amplification of desmin exons with intronic primers designed for this purpose (primer sequences are available on request). Amplification was carried out using an optimal procedure designed for each separate segment. PCR produced DNA fragments were purified using a QIAquick PCR Purification Kit (Qiagen) and directly sequenced using the DyeTerminator ${ }^{\mathrm{TM}}$ Sequencing Protocol (Perkin Elmer). In addition, the fragments amplified from genomic DNA were digested with restriction endonucleases RsaI and Bst NI (New England Biolabs) according to the manufacturer's instructions and resolved in a $4 \%$ NuSieve GTG $^{\circledR}$ low melting agarose gel (FMC BioProducts).

EXPRESSION OF GENOMIC DNA FRAGMENTS

CONTAINING SPLICE SITE MUTATIONS

Genomic DNA fragments comprising exons 2, 3, part of exon 4, and introns 2 and 3 were amplified from patients CSM- 6 and CSM-7/1 and a control's total genomic DNA using primers D2/4F (5'-TCACCCGCAACTGTC TGTCTGTCTTTCTGT-3') and D2/4R (5'AGACATGTCCATCTCCACCTG-3'). The resulting 502 bp DNA fragment was cloned into pCR2.1 vector (Invitrogen) and the entire sequence of each clone verified by sequencing. The EcoRI fragment of the pCR2.1 clone that included either the normal or the mutant desmin sequences of patients CSM6 and CSM$7 / 1$ were introduced into a mammalian expression vector pcDNA3.1 (Invitrogen) in the forward direction from exon 2 to exon 4 . SW13 (vim-) cells were transfected with these expression vectors and after 48 hours of incubation total RNA was extracted using RNeasy Kit (Qiagen). Potential contamination with DNA was excluded by incubation with RQ1 Rnase free DNase (Promega). RT-PCR was performed with Dnase treated total RNA using the Superscript RT protocol (Gibco BRL), followed by PCR with primers D2/4F and D2/4R used to produce the original DNA fragment. The transcripts were examined by electrophoresis in $4 \%$ NuSieve GTG low melting agarose gel (FMC BioProducts). An aliquot of each cDNA sample was cloned into the pCR2.1 plasmid; five clones of each transcript were selected and sequenced.

EXPRESSION OF THE MUTANT TRANSCRIPT IN SW13 (VIM-) CELLS

The mutant (patient CSM-6 smaller transcript) and control cDNA were cloned into the pCR2.1 plasmid (Invitrogen). Each construct was verified by sequence analysis. A HindIIIXhoI fragment enclosing either normal or mutant desmin cDNA was excised from clone pCR2.1 and ligated into a mammalian expression vector pcDNA3.1 (Invitrogen). The resulting constructs, $\mathrm{pDesH}$ containing the mutant desmin cDNA fragment from patient CSM-6 and pDesC that enclosed the wild type cDNA sequence from the control, were transfected into the SW13 (vim-) cells. The SW13 (vim-) cells do not normally express desmin, vimentin, or keratin, which makes them ideal for in vitro intermediate filament expression studies. Cells were grown to $50 \%$ confluence with DMEM supplemented with $10 \%$ fetal bovine serum and at this point transfected with $\mathrm{pDesH}$ and $\mathrm{pDesC}$ expression constructs, using Effectene Transfection Reagent (Qiagen), according to the manufacturer's protocol. At 48 hours after transfection, cells were washed three times with phosphate buffered saline (PBS) and fixed with 4\% paraformaldehyde for 15 minutes at room temperature. The cells were subsequently incubated with monoclonal antibody against human desmin (Sigma, D1033) for 16 hours at $4^{\circ} \mathrm{C}$ and treated with a secondary antibody conjugated with tetramethylrhodamine isothiocyanate (Sigma, T7657). After washing with PBS, the cells were analysed by immunofluorescence microscopy using a confocal microscope.

MICROSATELLITE GENOTYPING AND SINGLE NUCLEOTIDE POLYMORPHISM (SNP) ANALYSIS Methodology for paternity testing included genotyping with microsatellite markers D2S165, D3S1263, D4S404, D5S400, and D7S657.22 Their combined power for paternity exclusion was 0.998. PCR amplification was carried out using conditions recommended for each marker. The PCR amplified fragments were mixed with a size standard (ABI GS500 ROX) and loaded onto a $6 \%$ denaturing 
acrylamide gel. Electrophoresis was performed at $30 \mathrm{~W}$ for seven hours on an ABI 373A sequencer (Perkin Elmer). Data were collected and analysed using GS ABI 672 software (Perkin Elmer). The size of the amplified microsatellite alleles (in nucleotides) was determined and used in the analysis.

To determine which parent contributed the affected gamete, four single nucleotide polymorphisms (SNPs) at nucleotide positions $408,828,1014$, and 1104 of the desmin gene were tested in patient CSM- 6 and her parents. Three DNA polymorphisms previously identified in exons 4, 5, and 6 of the desmin gene ${ }^{182324}$ alter a restriction pattern and could be screened by digestion with endonucleases EcoRV, SmlI, and BssHII, respectively. The polymorphism at nucleotide 408 (Park et al, unpublished data) does not create a convenient restriction pattern and was screened by sequencing. The SNP haplotypes were constructed based on sequencing results and pedigree analysis.
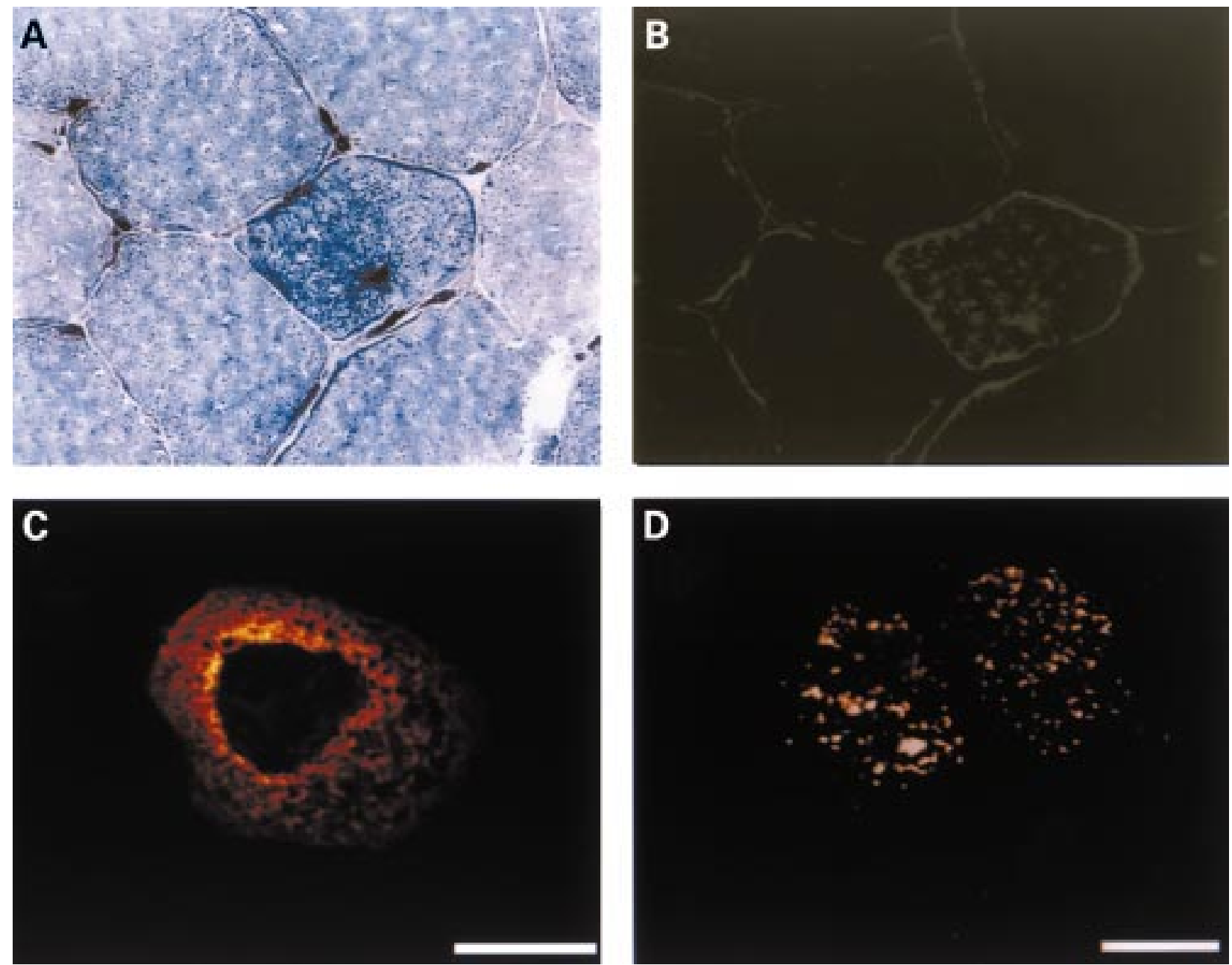

Figure 1 ( $A, B)$ Serial sections of a muscle biopsy specimen, patient CSM-6. (A) Modified Gomori trichrome staining. Cytoplasmic inclusions are seen in the perinuclear and subsarcolemmal areas. (B) Immunocytochemical analysis with fluorescein conjugated monoclonal antibodies against desmin (DACO, desmin D33). The intracytoplasmic inclusions show the presence of desmin immunoreactive material. (C, D) Functional analysis of the mutant desmin. Expression vectors containing either full length cDNA from the control or mutant desmin cDNA from patient CSM-6 were transfected into SW13 (vim-) cells. The cells were subsequently incubated with human desmin monoclonal antibody and a secondary antibody and analysed by immunofluorescence microscopy using a confocal microscope. (C) Cells transfected with a construct comprising a wild type desmin cDNA fragment show intense desmin positive material evenly distributed in the cytoplasm and particularly abundant in the perinuclear areas. (D) SW13 (vim-) cells transfected with a plasmid that contained mutant cDNA display a completely different pattern characterised by aggregation of desmin positive material into disrupted irregular clumps scattered throughout the cytoplasm. Bar: $20 \mu \mathrm{m}$.

\section{Results}

Patient CSM-6 experienced syncopal and fainting episodes at 38 years of age. A complete atrioventricular (AV) block was diagnosed and a permanent pacemaker was inserted at that time. At the age of 40, she developed slowly progressive weakness and atrophy in the legs, On examination (aged 48 years), the patient complained of energy loss, fatigue, progressive muscle weakness, and unsteadiness. Significant bilateral weakness and diffuse atrophy of the upper and lower extremities were observed, ness. Complete AV block was found on EKG. Muscle biopsy showed abundant intracytoplasmic inclusions in perinuclear and subsarcoleminternal nuclei, atrophic fibres, and cytoplasmic bodies were seen (fig 1A). The intracytoplasmic inclusions were strongly immunoreactive for desmin (fig 1B). All desmin positive vacuoles and small agregates of rods, multiple 
regions were also strongly positive for dystrophin and weakly positive for vimentin and spectrin. The patient is the only child and does not have children of her own. Neither the parents nor more distant relatives are known to have neuromuscular or cardiac disease.

Clinical and pathological data on family CSM-7 have been previously reported. ${ }^{25}$ The older of the two affected brothers, patient CSM-7/1, experienced dizziness at 29 years of age and at the age of 31 developed bradycardia and second degree $\mathrm{AV}$ block requiring insertion of a permanent pacemaker. He was later diagnosed as having cardiomyopathy with dilatation of the right ventricle, recurrent left sided cardiac failure, complete AV block, and pulmonary hypertension. Gait disturbance and weak-

A

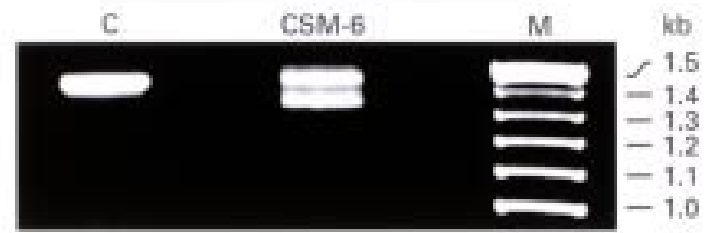

B

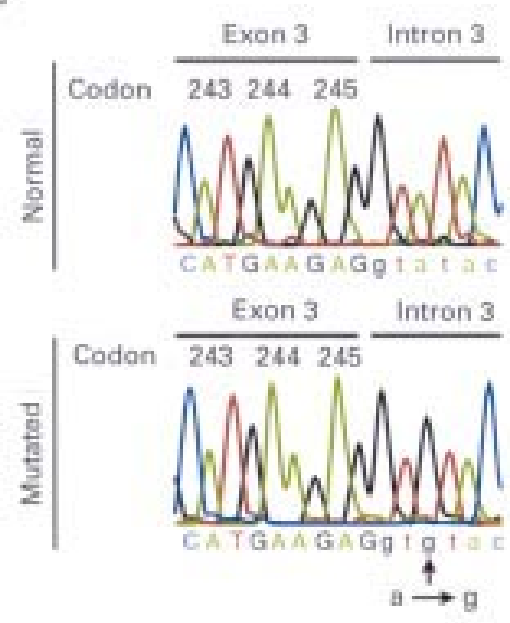

C
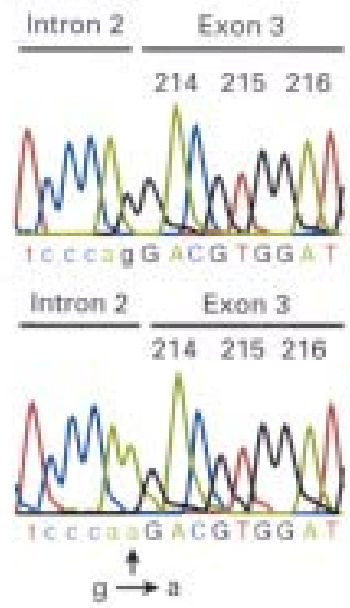

D

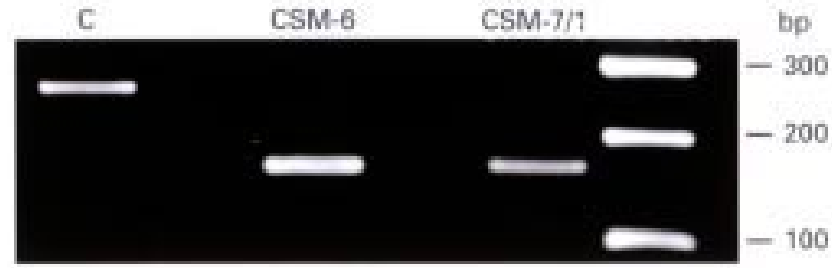

Figure 2 Identification of desmin splice variants. (A) Detection of two forms of desmin transcripts in the muscle biopsy tissue of patient CSM-6. Agarose gel analysis of the RT-PCR amplified products shows the presence of two transcripts (lane CSM-6), of which the larger corresponds to the control $1437 \mathrm{bp}$ amplification product (lane $C$ ) and the second band is about $100 \mathrm{bp}$ smaller. (B) Identification of an $A$ to $G$ substitution at the +3 position of the splice donor site flanking exon 3 in patient CSM-6 (IVS3+3A $\rightarrow G)$. (C) $G$ to $A$ transition at the -1 position of the splice acceptor site identified in patient CSM-7/1 $(I V S 2-1 G \rightarrow A)$. Arrows point to the identified alterations. (D) Expression of amplified genomic DNA fragments that contained the IVS3+3A $\rightarrow G$ and IVS2-1G $\rightarrow A$ mutations. Genomic DNA fragments were subcloned into a mammalian expression vector and transfected into the SW13 (vim-) cells. $m R N A$ extracted from the SW13 (vim-) cells was subjected to RT-PCR and the transcripts were analysed in an agarose gel and sequenced. Cells transfected with the control DNA produced a 259 bp transcript retaining exon 3 (lane C). Cells transfected with DNA fragments from patients CSM-6 and CSM-7 produced 163 bp transcripts with exon 3 missing (lanes CSM-6 and CSM-7). ness in the legs first appeared at 42 years and progressed to involve both hands. Examination at 50 years showed symmetrical weakness and atrophy in the hands and legs and bilateral pes cavus, but normal reflexes and senses. There were electromyographic signs of myopathy. A muscle biopsy was performed at this time. Many muscle fibres showed accumulation of granulofilamentous material located in subsarcolemmal areas, cytoplasmic bodies, and patch-like lesions immunoreactive with antibodies against desmin, alphaB-crystallin, and dystrophin. The patient died of cardiac insufficiency aged 52 years. Patient CSM-7/2 also developed a third degree AV block and required a pacemaker at the age of 41 , but had no muscle weakness when last examined aged 50 years. Their sister was unaffected. The parents died early and there is no information about distant relatives.

IDENTIFICATION OF SPLICE SITE MUTATIONS RT-PCR amplification of the mRNA extracted from muscle biopsy tissue of patient CSM-6 yielded a $1437 \mathrm{bp}$ cDNA product corresponding to the expected normal size. In addition, there was a faster migrating $1341 \mathrm{bp}$ fragment of about equal band intensity (fig $2 \mathrm{~A}$ ), indicating that two distinct desmin transcripts were present in muscle biopsy tissue of patient CSM-6, one of a normal length and another 96 bp smaller. Both transcripts were cloned and sequenced. Sequence analysis showed that the 1437 bp transcript from the control subject and the larger band from the patient were homologous to the normal cDNA sequence, while the patient's smaller $1341 \mathrm{bp}$ transcript lacked 96 internally deleted nucleotides, from 640 to 735 , according to the published desmin cDNA sequence (GenBank accession No AF055081). The deleted region matched the sequence of the entire exon 3, suggesting a possible splicing defect.

To define the molecular defects responsible for the exon 3 deletion, a $502 \mathrm{bp}$ DNA fragment comprising exons 2,3 , part of exon 4 , and introns 2 and 3 was amplified from genomic DNA, cloned, and sequenced. Analysis of the intron-exon boundaries flanking exon 3 allowed identification of an A to G substitution at the third nucleotide position of the intron 3 splice donor site in four of eight clones from patient CSM-6 (fig 2B), implying the presence of a splice site mutation $(\mathrm{IVS} 3+3 \mathrm{~A} \rightarrow \mathrm{G})$. A heterozygous $\mathrm{G}$ to $\mathrm{A}$ transition was identified at the -1 position of the intron 2 splice acceptor site of patient CSM7/1 (fig 2C), indicating the presence of a IVS2$1 \mathrm{G} \rightarrow \mathrm{A}$ mutation. The $\mathrm{AG}$ dinucleotide is highly conserved and its damage usually results in deletion of the downstream exon. Exon 3 spans $96 \mathrm{bp}$ of the DNA sequence that encodes 32 complete codons, from 214 to 245 . Deletion of exon 3 in desmin mRNA would remove 32 amino acids without interrupting the reading frame. The intronic IVS3+3AG mutation creates a restriction site for endonuclease RsaI, while the IVS2-1G $\rightarrow$ A mutation destroys a cleavage site for $B s t$ NI. To verify that these two mutations are not common splicing variants, 
we used restriction analysis to screen 150 healthy unrelated controls (300 chromosomes) from North American, European, and Asian populations. None of the controls carried the splice site mutations identified in our patients (data not shown), making it highly unlikely that they represent human polymorphisms.

EFFECTS OF SPLICE SITE MUTATIONS IN EXPRESSION SYSTEM

Fragments of amplified genomic DNA with the splice site mutations were expressed in SW13 (vim-) cells to determine whether each of the identified IVS $3+3 \mathrm{~A} \rightarrow \mathrm{G}$ and IVS $2-1 \mathrm{G} \rightarrow \mathrm{A}$ mutations actually cause exon 3 deletion. A $502 \mathrm{bp}$ fragment encompassing desmin exons 2, 3 , part of exon 4, and introns 2 and 3 was amplified from patients CSM-6 and CSM-7/1 and the control genomic DNA. Expression constructs that included these amplification products were transfected into the SW13 (vim-) cells. Subsequently, mRNA was extracted and transcripts were analysed in an agarose gel and sequenced. Cells transfected with constructs that included control DNA produced a normal length 259 bp transcript, suggesting correct splicing. In contrast, cells transfected with mutant DNA containing either the IVS $3+3 \mathrm{~A} \rightarrow \mathrm{G}$ or the IVS $2-1 \mathrm{G} \rightarrow \mathrm{A}$ mutation produced $163 \mathrm{bp}$ transcripts (fig $2 \mathrm{D})$. Sequencing showed that each of the aberrant $163 \mathrm{bp}$ transcripts was missing the exon 3 sequence. This result confirmed that the splice site mutations were responsible for exon 3 deletion in our patients.

FUNCTIONAL ANALYSIS OF MUTANT DESMIN

To investigate the functional consequences of desmin protein lacking 32 amino acids, expression vectors $\mathrm{pDesH}$ enclosing full length mutant cDNA from patient CSM-6 and $\mathrm{pDesC}$ containing wild type cDNA sequence from a control were transfected into SW13 (vim-) cells. The cell cultures were analysed by immunofluorescence microscopy. Cells transfected with $\mathrm{pDesC}$ plasmid that included desmin cDNA from a control produced fully functional desmin protein. It reacted positively with the desmin specific antibody and was evenly distributed in the cytoplasm with slightly more intense presence in the perinuclear areas, as expected of a normal intermediate filament network (fig 1C). Immunofluorescence analysis of the SW13 (vim-) cells transfected with the mutant construct shows completely disorganised coarse aggregates and clumps scattered throughout the cytoplasm (fig 1D).

THE ORIGIN OF THE MUTANT ALLELE IN THE CSM-6 FAMILY

The parents of patient CSM-6, carefully examined by two neurologists, and her more distant relatives do not show signs of myopathy or cardiac disease. To investigate the origin of the IVS $3+3 \mathrm{~A} \rightarrow \mathrm{G}$ mutation, we studied DNA samples from the mother and the father of patient CSM-6. Direct sequencing of PCR produced DNA fragments amplified from genomic DNA of both parents and restriction
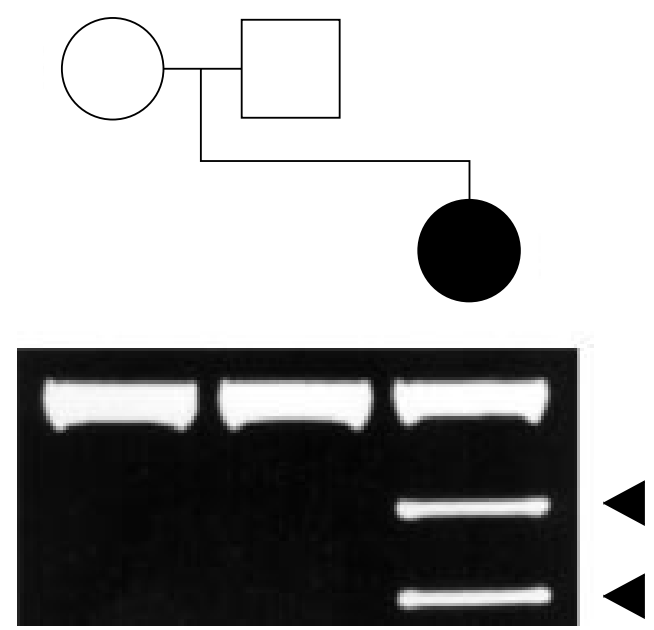

Figure 3 Family CSM-6 analysis. The IVS3 $+3 A \rightarrow G$ mutation changes restriction pattern for endonuclease RsaI that permits screening for this mutation. The patient shows a three band pattern suggesting the presence of the mutation (arrowheads point to the bands associated with the mutation). Both unaffected parents lack this pattern. The absence of the IVS $3+3 A \rightarrow G$ mutation in the parents was confirmed by sequencing.

Table 1 Microsatellite alleles (measured in $b p$ ) in members of family CSM-6

\begin{tabular}{lllllll}
\hline Marker & Mother & & Father & \multicolumn{3}{c}{ Patient } \\
\hline D2S165 & 152 & 160 & 142 & 158 & 158 & 160 \\
D3S1263 & 197 & 209 & 183 & 205 & 183 & 209 \\
D4S405 & 284 & 294 & 292 & 292 & 284 & 292 \\
D5S400 & 230 & 264 & 226 & 230 & 230 & 230 \\
D7S657 & 252 & 266 & 262 & 266 & 252 & 266 \\
\hline
\end{tabular}

Table 2 Intragenic single nucleotide polymorphism (SNP) haplotypes in the desmin myopathy patient and her parents

\begin{tabular}{|c|c|c|c|c|c|c|}
\hline \multirow{2}{*}{$\frac{\text { Nucleotide No* }}{408}$} & \multicolumn{2}{|c|}{ Mother } & \multicolumn{2}{|c|}{ Father } & \multicolumn{2}{|c|}{ Patient } \\
\hline & $\mathrm{C}$ & $\mathrm{T}$ & $\underline{\mathrm{C}}$ & $\mathrm{C}$ & $\underline{\mathrm{C}}$ & $\mathrm{T}$ \\
\hline 828 & C & C & $\overline{\mathrm{T}}$ & C & $\overline{\mathrm{T}}$ & C \\
\hline 1014 & G & G & $\overline{\mathrm{C}}$ & G & $\underline{\overline{\mathrm{C}}}$ & G \\
\hline 1104 & G & G & $\overline{\mathrm{A}}$ & G & $\overline{\mathrm{A}}$ & G \\
\hline $\mathrm{A} \rightarrow \mathrm{G}$ mutation & A & A & $\underline{\overline{\mathrm{A}}}$ & A & $\overline{\bar{G}}$ & A \\
\hline
\end{tabular}

*Nucleotide numbers correspond to the GenBank desmin cDNA sequence (AF055081).

Underlined: paternal SNP haplotype, on which the A to G transition has occurred.

analysis with RsaI endonuclease (fig 3) failed to detect this mutation. To trace the origin of the mutant allele, microsatellite genotyping, and intragenic single nucleotide polymorphism (SNP) analysis were performed. The results exclude alternative paternity, based on analysis of inheritance of the microsatellite marker alleles (table 1) and SNP haplotypes (table 2). The father and daughter share a CTCA haplotype that carries the A to $G$ substitution in the daughter, but not in the father (table 2). This analysis conclusively shows that the mutation has occurred de novo on the father's CTCA haplotype.

\section{Discussion}

This study has identified splice site mutations in the desmin gene that result in deletion of 32 amino acids from the desmin protein and cause deleterious effects in the cardiac and skeletal muscles of these patients as well as in the SW13 (vim-) cells transfected with the mutant allele. 
Exon skipping is a relatively common finding in inherited diseases often caused by point mutations that inactivate consensus splice sites. ${ }^{26}$ The highly conserved AG splice acceptor and GT splice donor sites are the most frequent targets of mutations affecting splicing, ${ }^{27}$ but many mutations occur at less stringently conserved positions of the consensus splice junctions flanking the invariant $A G$ and GT. ${ }^{27}{ }^{28}$ The nucleotide at position +3 of the splice donor site that was mutated in patient CSM-6 is the least conserved. Only about $49 \%$ of splice donor sites have an $\mathrm{A}$ at this position. Analysis of 1801 native splice donor sites indicated that the sites with a $G$ at the +3 position have +4 to +6 nucleotides complementary to the end sequence of the U1 snRNA that is responsible for splice donor site recognition. ${ }^{29}$ In the desmin exon 3 splice donor site, the wild type sequence of nucleotides at positions +4 to +6 does not match the U1 snRNA sequence in any of the three nucleotide positions. Therefore, according to this theory, the $A \rightarrow G$ mutation at +3 position would be incompatible with normal splicing. The IVS2 $-1 \mathrm{G} \rightarrow \mathrm{A}$ mutation identified in our second patient changes the highly conserved canonical AG splice acceptor site, strongly suggesting the presence of a splicing defect.

Several lines of evidence indicate that the splice site mutations were responsible for the cardiac and skeletal myopathy in our patients. By expressing genomic DNA fragments that included IVS2 $-1 \mathrm{G} \rightarrow \mathrm{A}$ and IVS3 $+3 \mathrm{~A} \rightarrow \mathrm{G}$ mutations, we showed that these mutations inactivate splice sites and are responsible for the deletion of exon 3. The 96 bp exon 3 sequence encodes 32 complete codons; therefore an internal deletion of exon 3 would not interrupt the reading frame and is predicted to result in synthesis of a desmin polypeptide that is 32 amino acids smaller than normal. Expression studies in the SW13 (vim-) cells indicated that the mutant desmin is expressed but is not functional. Unlike the cells transfected with cDNA from the control, cells containing mutant cDNA showed disrupted shapeless aggregates and clumps of desmin positive material similar to disorganised desmin inclusions in the patients' affected muscles.

The disease in patient CSM-6 is sporadic since both her parents were unaffected and more distant family members were not known to have myopathy. Previously published reports on desmin related myopathies include 22 families with autosomal dominant inheritance, seven families with a possible autosomal recessive pattern, a single family with an established compound heterozygosity, and 23 patients with no family history. Possible explanations for the occurrence of sporadic cases of a disease known to be inherited could be nonpenetrance, non-Mendelian inheritance, false paternity, presence of hot spot regions for mutations, or germline mosaicism. Maternity and paternity were unequivocally confirmed in the CSM- 6 family, but neither parent carried the IVS $3+3 \mathrm{~A} \rightarrow \mathrm{G}$ mutation and no hot spot regions for somatic mutations in the desmin gene have yet been identified. According to the results of the haplotype analysis performed by using intragenic SNPs, the mutation has occurred de novo on the father's allele. It is likely that in this case the father was a germline mosaic.

This study provides evidence that splice site mutations in the desmin gene resulting in skipping of exon 3 during the pre-mRNA splicing are responsible for familial and sporadic myopathy. Molecular studies of desmin myopathy add to better understanding of this heterogeneous group of neuromuscular disorders and permit a precise molecular diagnosis of this disorder, thereby facilitating prenatal testing and attempts at gene therapy.

The authors are grateful to the members of the affected families for their enthusiastic participation in this study. GenBank accession numbers of sequences generated in this study: patient CSM-6, AF189280; patient CSM-7/1, AF189281.

1 Goebel HH. Desmin-related neuromuscular disorders. Muscle Nerve 1995;18:1306-20.

2 Goebel HH. Desmin-related myopathies. Curr Opin Neurol 1997;10:426-9.

3 Porte A, Stoeckel ME, Sacrez A, Batzenschlager A. Unusual familial cardiomyopathy with storage of intermediate filaments in the cardiac muscular cells. Virchows Arch A 1980;386:43-58.

4 Nakano S, Engel AG, Waclawik AJ, Emslie-Smith AM, Busis NA. Myofibrillar myopathy with abnormal foci of desmin positivity. 1. Light and electron microscopy analysis of 10 cases. F Neuropathol Exp Neurol 1996;55:549-62.

5 De Bleecker JL, Engel AG, Ertl BB. Myofibrillar myopathy with abnormal foci of desmin positivity. 2 . Immunocytochemical analysis reveals accumulation of multiple other proteins. F Neuropathol Exp Neurol 1996;55:563-77.

6 Lazarides E. Intermediate filaments as mechanical integrators of cellular space. Nature 1980;238:249-56.

7 Geisler N, Kaufmann E, Weber K. Proteinchemical characGeisler N, Kaufmann E, Weber K. Proteinchemical charac-
terization of three structurally distinct domains along the terization of three structurally distinct domains along the
protofilament unit of desmin $10 \mathrm{~nm}$ filaments. Cell protofilament un

8 Fuchs E, Weber K. Intermediate filaments: structure, dynamics, function, and disease. Annu Rev Biochem 1994;63:345-82.

9 Geisler N, Weber K. The amino acid sequence of chicken muscle desmin provides a common structural model for intermediate filament proteins. EMBO F 1982;1:164956.

10 Viegas-Péquignot E, Li Z, Dutrillaux B, Apiou F, Paulin D. Assignment of human desmin gene to band 2q35 by non-radioactive in situ hybridization. Hum Genet 1989;83:

1 Li Z, Lilienbaum A, Butler-Browne G, Paulin D. Human desmin-coding gene: complete nucleotide sequence, characterization and regulation of expression during myogenacterization and regulation of expression durin
esis and development. Gene 1989;78:243-54

12 Capetanaki Y, Milner DJ, Weitzer G. Desmin in muscle forCapetanaki Y, Milner DJ, Weitzer G. Desmin in muscle for-
mation and maintenance: knockouts and consequences. Cell Struct Funct 1997;22:103-16.

13 Li Z, Colucci-Guyon E, Pincot-Raymond M, Mericskay M, Pournin S, Paulin D, Babinet C. Cardiovascular lesions and skeletal myopathy in mice lacking desmin. Dev Biol 1996;175:362-6.

14 Thornell LE, Carlsson L, Li Z, Mericskay M, Paulin D. Null mutation in the desmin gene gives rise to a cardiomyopathy. $\mathcal{F}$ Mol Cell Cardiol 1997;29:2107-24.

15 Raats JMH, Henderik JBJ, Verdijk M, van Oort FLG, Gerards WLH, Ramaekers FCS, Bloemendal H. Assembly of carboxy-terminally deleted desmin in vimentin-free cells. Eur F Cell Biol 1991;56:84-103.

16 Yu KR, Hijikata T, Lin ZX, Sweeny HL, Englander SW, Holtzer H. Truncated desmin in PtK2 cells induces Holtzer $H$. Truncated desmin in PtK2 cells induces
desmin-vimentin-cytokeratin coprecipitation, involution of desmin-vimentin-cytokeratin coprecipitation, involution of
intermediate filament networks, and nuclear intermediate filament networks, and nuclear Pragmentation: a model for many degener

17 Raats JM, Schaart G, Henderik JB, van der Kemp A, Dunia I, Benedetti EL, Pieper FR, Ramaekers FC, Bloemendal H. Muscle-specific expression of a dominant negative desmin mutant in transgenic mice. Eur $\mathcal{F}$ Cell Biol 1996; 71:221-36.

18 Goldfarb LG, Park KY, Cervenakova L, Gorokhova S, Lee $\mathrm{K}$, Dalakas MC. Missense mutations in desmin associated with familial cardiac and skeletal myopathy. Nat Genet 1998;19:402-3.

19 Munoz-Marmol AM, Strasser G, Isamat M, Coulombe PA, Yang Y, Roca X, Vela E, Mate JL, Coll J, FernandezYang Y, Roca X, Vela E, Mate JL, Coll J, Fernandez-
Figueras MT, Navas-Palacios JJ, Ariza A, Fuchs E. A dysfunctional desmin mutation in a patient with severe generfunctional desmin mutation in a patient with severe gener17. 
20 Li D, Tapscoft T, Gonzalez O, Burch PE, Quinones MA, Zoghbi WA, Hill R, Bachinski LL, Mann DL, Roberts R. Desmin mutation responsible for idiopathic dilated cardiomyopathy. Circulation 1999;100:461-4.

21 Sjoberg G, Saavedra-Matiz CA, Rosen DR, Wijsman EM, Borg K, Horowitz SH, Sejersen T. A missense mutation in the desmin rod domain is associated with autosomal dominant distal myopathy, and exerts a dominant negative effect on filament formation. Hum Mol Genet 1999;8:2191-8.

22 Ohno Y, Sebetan IM, Akaishi S. A simple method for calculating the probability of excluding paternity with any number of codominant alleles. Forensic Sci Int 1982;19:93-8

23 Shaw MA, Atkinson SE, Li Z, Paulin D, Blackwell JM. EcoRV RFLP of the desmin (DES) gene and MspI RFLP of the villin (VIL1) gene on human chromosome 2. Hum Mol Genet 1992;1:448.

24 Vicart P, Dupret JM, Hazan J, Li Z, Gyapay G, Krishnamoorthy R, Weissenbach J, Fardeau M, Paulin D. Human desmin gene: cDNA sequence, regional localization and exclusion of the locus in a familial desmin-related myopathy. Hum Genet 1996;98:422-9.
25 Goebel HH, Voit T, Warlo I, Jacobs K, Johannsen U, Muller CR. Immunohistologic and electron microscopic abnormalities of desmin and dystrophin in familial cardiomyopathy and myopathy. Rev Neurol 1994;150:452-9.

26 Nakai K, Sakamoto H. Construction of a novel database containing aberrant splicing mutations of mammalian genes. Gene 1994;141:171-7.

27 Krawczak M, Reiss J, Cooper DN. The mutational spectrum of single base-pair substitutions in mRNA splice junctions of human genes: causes and consequences. Hum Genet 1992;90:41-5.

28 Padgett RA, Grabowski PJ, Konarska MM, Seiler S, Sharp PA. Splicing of messenger RNA precursors. Annu Rev Biochem 1986;55:1119-50.

29 Ohno K, Brengman JM, Felice KJ, Cornblath DR, Engel AG. Congenital end-plate acetylcholinesterase deficiency caused by a nonsense mutation and an $\mathrm{A} \rightarrow \mathrm{G}$ splice-donorsite mutation at position +3 of the collagenlike-tail-subunit gene $(C O L O)$ : how does $\mathrm{G}$ at position +3 result in aberrant splicing? Am f Hum Genet 1999;65:635-44. 